\title{
Natura@economía
}

ISSN 2226-9479 (Versión electrónica) Website: http://revistas.lamolina.edu.pe/index.php/neu

\section{Análisis de la cadena agroproductiva de cebada (Hordeum vulgare) del Carchi y frutilla (Fragaria sp) en Pichincha}

\section{Analysis of the agricultural production chain of barley (Hordeum vulgare) from Carchi and strawberry (Fragaria $s p$ ) in Pichincha}

\author{
Juan Pablo Aragón ${ }^{1 *}$; Elisandra Parra ${ }^{1}$; Ángel Chicaiza ${ }^{1}$; Mauricio Aragón ${ }^{1}$
}

\begin{abstract}
${ }^{1}$ Facultad de Ingeniería en Ciencias Agropecuaria y Ambientales, Universidad Técnica del Norte, Ibarra, Ecuador. Email: jparagon@utne.edu.ec; m_aragon88@yahoo.es; ely.221_pcj@hotmail.com; john_21b@hotmail.com
\end{abstract}

Recepción: 02/10/2019; Aceptación: 15/12/2019

\section{Resumen}

El objetivo del presente estudio fue analizar la cadena agroproductiva de cebada (Hordeum vulgare) del Carchi y frutilla (Fragaria sp) en Pichincha. El estudio planteó una investigación descriptiva a través de un método deductivo e inductivo para establecer variables y cuantificarlas mediante la técnica de encuesta y entrevista a productores y comerciantes. El estudio determinó la cadena agroproductiva de la cebada y de la frutilla planteando las mejores estrategias para mejorar los ingresos del agricultor. En conclusión, un cultivo tradicional es reemplazado por un no tradicional por generar mayor utilidad, tener proyección de un mercado externo y no tener en la superficie de siembra.

Palabras clave: cultivos tradicionales; cadena agroproductiva; utilidad; estrategias.

\begin{abstract}
The objective of the present study was to analyze the agricultural chain of barley (Hordeum vulgare) from Carchi and strawberry (Fragaria sp) in Pichincha. The study proposed a descriptive investigation through a deductive and inductive method to establish variables and quantify them using the technique of survey and interview with producers and merchants. The study determined the agricultural production chain of barley and strawberries, proposing the best strategies to improve the farmer's income. In conclusion, a traditional crop is replaced by a non-traditional one because it generates greater utility, has a projection of an external market and does not have on the planting surface.
\end{abstract}

Keywords: traditional crops; agroproductive chain; utility; strategies.

Forma de citar el artículo: Aragón et al., 2019. El Análisis de la cadena agroproductiva de cebada (Hordeum vulgare) del Carchi y frutilla (Fragaria sp) en Pichincha. Natura@economía 4(2): 70-76 (2019). 


\section{Introducción}

Ecuador es un país eminentemente agrícola, los ingresos generados por esta actividad son aproximadamente 5 mil millones de USD que representaron el $7 \%$ del PIB nacional en el año 2017 (Ministerio de Agricultura, Ganadería, Acuacultura y Pesca, 2017). Los principales cultivos en el país son: banano (Mussa paradisiaca), cacao (Teobroma cacao), café (Coffea arábiga), papa (Solanum tuberosum), maíz (Zea mays), fréjol (Phaseolus vulgaris), arroz (Oryza sativa), trigo (Tritucum $s p$ ), cebada (Hordeum vulgare) y rosas (Rosa $s p$ ), entre los principales. Varios de estos cultivos se los considera como tradicionales por su importancia económica; por ser cultivos ancestrales o por su relevancia en la seguridad alimentaria (Monteros y Salvador, 2015). En el país existen cultivos no tradicionales como el mango (Mangifera indica), kiwi (Actinidia deliciosa) y frutilla (Fragaria sp), sin embargo, su importancia económica se ha incrementado en las tres últimas décadas, especialmente la frutilla en las provincias de Imbabura y Pichincha.

Las cadenas de comercialización de productos agrícolas no se encuentran identificadas en Ecuador, menos aún en cultivos no tradicionales o de baja importancia económica, es el caso de la frutilla y la cebada respectivamente. Esto genera que no se optimicen procesos de comercialización reduciendo la utilidad de los productores, por ende sus niveles de vida. Es prioritario identificar eslabones, márgenes de comercialización y mercados para plantear estrategias que mejoren los ingresos de los agricultores. Por ello, el objetivo principal de la investigación fue analizar las cadenas agroproductivas de un cultivo tradicional (cebada) y no tradicional (frutilla).

\section{Materiales y métodos}

La investigación se la realizó en la provincia del Carchi, cuya superficie es $3604,33 \mathrm{Km}^{2}$, se encuentra situada entre los 1000 y 4,723 msnm. Su población alcanza un total de 164,524 habitantes que representan el 1,1\% de la población total del Ecuador $(81,155$ hombres y 83,369 mujeres) 82,495 sector urbano y 82,029 sector rural (Prefectura del Carchi, 2016); y, en la provincia de
Pichincha, parroquia de Yaruquí, a una altitud promedio de 2,527 msnm, con temperaturas desde los 12 a $28{ }^{\circ} \mathrm{C}$, con precipitación anual dependiendo del piso ecológico entre $500 \mathrm{~mm}$ a $2000 \mathrm{~mm}$ anual. Este sector es donde se concentra la mayor producción de frutilla (Sistema de Investigación sobre la Problemática Agraria, 2013).

De acuerdo con Hernández y Fernández (2010) la investigación tiene un enfoque cuantitativo, es estructurada y necesita de una recolección de datos previa, por otra parte, una investigación de enfoque cualitativo toma forma en el desarrollo del trabajo de campo o realización del estudio no se rige a estructuras su diseño es flexible.

Se utilizó un método deductivo e inductivo para identificar la cadena de comercialización actual y determinar estrategias de comercialización.

Para la identificar los eslabones de la cadena de comercialización en frutilla se aplicó una encuesta estructurada a 75 productores, 14 comerciantes entre transportistas, mayoristas y minoristas. Se obtuvo datos sobre formas de presentación de la fruta para la venta, precios del producto, ubicación de los principales mercados e identificó a los actores que intervienen en la cadena de comercialización. En el caso de cebada, se encuestó a 125 productores y 10 comerciantes e industrializadores de la cebada para definir los mismos indicadores.

La determinación de estrategias óptimas de comercialización de frutilla y cebada se realizó mediante un análisis de factores externos e internos (FODA) y con el cruce de variables.

\section{Resultados y discusión}

Se determinó la productividad, rendimiento y los costos de producción de cebada en la provincia del Carchi. Son 902,5 ha en toda la provincia del Carchi. Sin embargo según el Instituto Nacional de estadísticas y censos en la Encuesta De Superficie y Producción Agropecuaria Continua (ESPAC, 2017), la superficie sembrada en la provincia del Carchi es de 1060 ha. Los costos de producción se estableció en la Tabla 1. Además, el rendimiento promedio de la cebada es de $65 \mathrm{qq} / \mathrm{ha}$, con un costo promedio de $598 \mathrm{USD} / \mathrm{ha}$, se tiene un costo/ 
Tabla 1: Costos de Producción de cebada por hectárea en la provincia del Carchi

\begin{tabular}{lrrrrrr}
\hline Actividades & \multicolumn{2}{c}{ Tradicional } & \multicolumn{3}{c}{ Semi-tecnificado } & \multicolumn{2}{c}{ Tecnificado } \\
\cline { 2 - 7 } & USD & $\%$ & USD & $\%$ & USD & $\%$ \\
\hline Análisis de suelo & 0,00 & 0,00 & 0,00 & 0,00 & 0,00 & 0,00 \\
Semilla & 64.00 & 13,21 & 59,24 & 10,40 & 58,89 & 9,82 \\
Siembra & 24,50 & 5,06 & 28,59 & 5,02 & 23,64 & 3,94 \\
Preparación del terreno & 75,00 & 15,48 & 82,60 & 14,50 & 118,72 & 19,79 \\
Fertilizantes & 87,50 & 18,06 & 123,47 & 21,68 & 145,81 & 24,31 \\
Sanidad & 26,00 & 5,37 & 58,74 & 10,31 & 60,89 & 10,15 \\
Riego & 0,00 & 0,00 & 29,25 & 5,14 & 19,64 & 3,27 \\
Cosecha & 120,00 & 24,77 & 139,57 & 24,51 & 132,67 & 22,12 \\
Transporte & 42,50 & 8,77 & 24,72 & 4,34 & 23,06 & 3,84 \\
Post - cosecha & 45,00 & 9,29 & 23,29 & 4,09 & 16,53 & 2,76 \\
Total & 484,50 & 100,00 & 569,47 & 100,00 & 599,83 & 100,00 \\
\hline
\end{tabular}

qq de 9,20 USD. En el mercado, un precio promedio de 18/qq, genera una utilidad del $50 \%$ sobre el precio de venta. Sin embargo, la falta de planificación en las siembras aplica la ley de oferta y demanda, llegando a un precio hasta $13 \mathrm{USD} / \mathrm{qq}$ que beneficia únicamente al productor en siembras por volumen sobre las 10 ha. En la provincia del Carchi el 57\% de productores de cebada tienen menos de 5 ha, esto implica que en un ciclo de cultivo de cinco a seis meses tienen una utilidad del $70 \%$ equivalente a un salario básico unificado por mes.

La cadena de comercialización de la cebada en la provincia del Carchi está definida: 1) Canal Indirecto Intermediario, el $66 \%$ de los agricultores vende la producción de cebada al intermediario en pie de finca y el $3 \%$ entrega su producto a centros de acopio. 2) Canal Directo: el 31\% de los productores utilizan este canal, buscando obtener mayores réditos, vende su producto en molinos, bodegas y centros de acopio. Los márgenes de comercialización se presenta en la Tabla 2.
En el cultivo de frutilla la distribución de la superficie de siembra es: 22 productores cultivan en extensiones que van desde los 4000 a $6000 \mathrm{~m}^{2}$, representa el $29 \%$, por otro lado, el $28 \%$ corresponde a cultivos de 1 ha, de modo que la producción total que abastece el mercado proviene de pequeños productores. En cuanto a los costos de producción según la superficie de siembra se detalla en la Figura 1.

Se presentan fluctuaciones en el costo de acuerdo a la inversión inicial y costo por mantenimiento, para extensiones de 7000 a $9000 \mathrm{~m}^{2}$ tiene un costo de $\$ 16575$ este monto es superior al costo de una hectárea de terreno que es de $\$ 15138$, se debe a que la adquisición de plántulas van desde los 0,20 a 0,25 centavos de igual forma en los demás insumos para la producción, en donde se aplica economías de escala. El rendimiento es de $708 \mathrm{~kg} / \mathrm{ha} / \mathrm{d}$, la cual se comercializa de acuerdo a la calidad, sin embargo, los productores realizan empíricamente la clasificación de la fruta con las siguientes denominaciones: de primera o clase extra,

Tabla 2: Margen Bruto de comercialización de cebada (productor-intermediario)

\begin{tabular}{lccc}
\hline \multicolumn{1}{c}{ Eslabón } & $\begin{array}{c}\text { Precio de venta del } \\
\text { Agricultor qq/USD }\end{array}$ & $\begin{array}{c}\text { Precio de venta al } \\
\text { Consumidor qq/usd }\end{array}$ & $\begin{array}{c}\text { Margen de } \\
\text { comercialización \% }\end{array}$ \\
\hline Intermediario & 16,3 & 19 & 14 \\
Centro de acopio & 13,0 & 21 & 38 \\
Industria & 19,9 & 80 & 75 \\
\hline
\end{tabular}




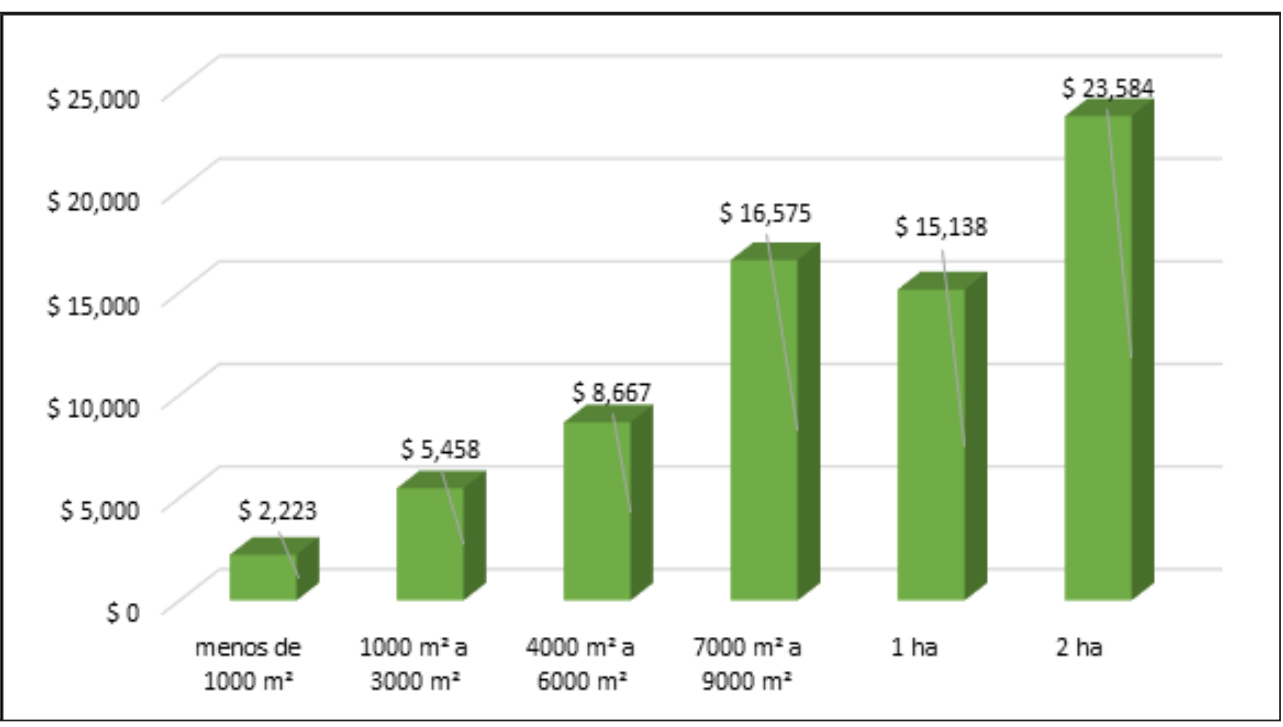

Figura 1: Costo de producción del cultivo de frutilla según la extensión de terreno

de acuerdo con los datos recopilados un 64\% de producción es de primera, esta categoría presenta un tamaño de 4 a $5 \mathrm{~cm}$ y color rojo intenso; el $27 \%$ corresponde a fruta de segunda o mediana y el $9 \%$ representa la producción de tercera clase. En la Tabla 3 se resume el precio de comercialización de la frutilla de primera clase.

Tabla 3: Precios oficiales referenciales de frutilla, mes de diciembre 2017

\begin{tabular}{|c|c|c|}
\hline $\begin{array}{l}\text { Mercado } \\
\text { Mayorista }\end{array}$ & $\begin{array}{c}\text { Balde } \\
\text { (12 Kilos) } \\
1^{\circ} \text { clase/ } \\
\text { USD } \\
\end{array}$ & Kilo/ USD \\
\hline Quito MMQ-EP & 17,70 & 1,55 \\
\hline Guayaquil-TTV & 27,88 & 2,04 \\
\hline
\end{tabular}

Tambien, se presenta fluctuación en los precios a nivel mayorista, debido a la participación de intermediarios quienes son los encargados de llevar el producto hasta estos mercados. De la frutilla proveniente de Yaruquí, el 63\% vende su producto a intermediarios, nueve son los comerciantes que adquieren la frutilla directamente a 47 productores; el $13 \%$ se entrega a supermercados como Supermaxi, Aki y
Santa María de localidades cercanas como Tumbaco, Cumbayá, Cayambe y Sangolquí; un $9 \%$ vende de forma directa, ya sea en el mercado minorista de la misma parroquia o en los mercados de parroquias cercanas; $7 \%$ entrega en los mercados mayoristas municipales (Mercado mayorista de Quito, Santo Domingo); un 5\% entregada a pequeñas y grandes industrias locales (PRONACA, Nuestra Huerta, Paraíso, Florella); y el 3\% vende su producción en ferias de forma eventual.

Según el cálculo del margen de comercialización con respecto al precio de la frutilla de clase extra o primera y, los eslabones representan al canal más utilizado por los agricultores de la parroquia de Yaruquí. Se ratifica que el productor es el menos beneficiado por la utilidad generada en la cadena agroproductiva.

Se han determinado dos estrategias óptimas para la comercialización de frutilla de Yaruquí. 1) La Asociatividad de los productores de la parroquia es clave para comercializar en volumen, manejar precios de mercado, buscar clientes estables que manejen precios justos, ser más competitivos en otros nichos, disminuir los eslabones en la cadena, todo esto con la finalidad de que el productor sea el más beneficiado. Con una asociación establecida se pueden incluir productores de todo el país y formar 
una sociedad fuerte como la de floricultores y bananeros. De la estrategia uno se deriva la exportación. Se ha identificado dos mercados potenciales para la frutilla, siendo estos Alemania y Reino Unido, para lo cual se recomienda realizar una investigación de mercado que ratifique estos nichos.

En la Provincia del Carchi, se ha generado cerca del $10 \%$ de baja en la productividad de cebada por la sustitución de los cultivos tradicionales por no tradicionales más rentables. Esto lo sustenta Cangas (2018) quien señala que, los agricultores en los dos últimos años abandonaron el cultivo del cereal por la falta de mercados; asimismo, retomaron el cultivo de papa y quinua, siendo el primero un cultivo tradicional y de importancia económica en la provincia y para el productor respectivamente. Esto significa que el cultivo de cebada no es rentable a los agricultores por la superficie de siembra .

Flores y Edwin (2017), informan que los cultivos tradicionales han dejado de ser económicamente rentables por la sobreproducción, importación del producto y porque se han implantado cultivos más rentables (los no tradicionales).

Iglesias (2017), en su estudio de comercialización de cebada en Argentina menciona que la cebada tiene canales de comercio cortas porque la mayoría se destina a la industria de malta y cerveza por ser un producto de primera necesidad; además este país, es el principal productor de cebada de la región y realiza exportaciones a diferentes países del mundo incluyendo a Ecuador, es decir, maneja la economía de este producto porque tienen una fortaleza, la asociatividad. En Ecuador, existe similar situación pero únicamente con el cultivo de banano al ser un cultivo tradicional y que cuenta con una organización muy fuerte a nivel nacional y mundial.

Como en la mayoría de las cadenas de comercialización de los productos agrícolas el margen de comercio más amplio se encuentra en los eslabones intermedios entre productor y consumidor final, siendo el primer eslabón el más perjudicado en la utilidad; además de ser el que mayor riesgo tiene al dedicarse a la producción que depende mayormente de factores externos como el clima y el mercado.
Las estrategias de comercialización definidas fueron: Incentivar la formación de asociaciones de productores; incentivar al mejoramiento de industrialización de la cebada; incursionar en los mercados de ensilaje, este último es de mayor aceptación por generar la rentabilidad más alta.

En referencia a la frutilla, Agronegocios (2010) publica que, la provincia de Pichincha es donde se encuentra la mayor cantidad de producción de frutilla a nivel nacional, con aproximadamente $400 \mathrm{~h}$; siendo la parroquia Yaruquí la de mayor producción y se caracteriza por tener fincas menores a 1 ha. Según el Sistema de Investigación sobre la Problemática Agraria (2013), son considerados pequeños productores aquellas UPAs con una extensión menor a 5 ha; esto repercute tanto en el rendimiento y costos de producción de las plantaciones frutilleras.

Para Tustón (2012) los parámetros y valores a considerar para la implementación del cultivo de frutilla varían de acuerdo al grado de tecnificación de la plantación, en la Figura 1 se detalla los costos de producción del cultivo de frutilla según la superficie de terreno. De acuerdo con de Instituto Interamericano de Cooperación para la Agricultura (IICA, 2017), la calidad de la frutilla considera aspectos tamaño, color, brillo y dureza; también se tiene en cuenta la presencia de enfermedades. Estos aspectos son considerados al clasificar la fruta de Yaruquí.

El $63 \%$ de la producción se llevan intermediarios que comercializan la fruta en la costa ecuatoriana, así lo ratifica Tustón (2012) "la producción de frutilla de la provincia de Pichincha tiene como destino los mercados de Esmeraldas, Guayaquil, Manta y Cuenca". La demanda de la fruta es tan amplia que existe déficit en la producción y se debe compensar con la producción de provincias aledañas como Imbabura. En tal virtud la cadena de comercio de la frutilla es tan compleja que solamente existen cadenas largas de comercio de los cuales se crean márgenes de comercialización amplios para los intermediarios que se detallan en la Tabla 4.

La cebada es un cultivo tradicional, no es rentable en superficies menores a 10 ha considerado su ciclo de cultivo, además, su producción está decreciendo lo que 
significa que no existe demanda. La cadena de comercio es relativamente corta de tres a cuatro eslabones que se pierde en la provincia de origen sin alternativa de un mercado externo. Por el contrario, la frutilla como cultivo no tradicional tiene mayores oportunidades, superficies menores a una ha son rentables, el retorno de capital es más rápido ya que su ciclo de 80 a 90 días hasta la producción que permite recobrar la inversión en menos tiempo. La cadena de comercio tiene 5 a 6 eslabones que incrementa el precio de venta afectando la utilidad del productor. Además la producción llega hasta provincias como Guayas y Azuay lo que significa que la demanda cada vez es mayor, incluso con altas expectativas de exportación.

Tabla 4: Márgenes de comercialización de la frutilla de Yaruquí

\begin{tabular}{lcc}
\hline Eslabón & $\begin{array}{c}\text { Precio de } \\
\text { venta/lb } \\
\text { Frutilla } \\
\text { clase extra }\end{array}$ & $\begin{array}{c}\text { Margen bruto de } \\
\text { comercialización } \\
\%\end{array}$ \\
\hline Productor & 0,60 & 7 \\
$\begin{array}{l}\text { Intermediario } \\
\text { Mercado }\end{array}$ & 0,72 & 10 \\
$\begin{array}{l}\text { Minorista } \\
\text { Consumidor }\end{array}$ & 0,80 & 20 \\
Final & 1,00 & 63 \\
\hline
\end{tabular}

Luna-Morales (2015), menciona que los cereales tradicionales de una región sirven fundamentalmente para cubrir la demanda alimenticia del sector y, su redituabilidad está en el mercado nacional. En cambio Vaquiro (2016), menciona que los cultivos no tradicionales buscan una alternativa económica a través de la sustitución de cultivos tradicionales. Los nuevos cultivos generan mayor rentabilidad por ser destinados a mercados externos.

Tanto para un cultivo tradicional como uno no tradicional la planificación en la siembra es un factor determinante en el precio al momento de la comercialización, esto se puede manejar bajo la organización de agricultores. Por tal razón, la asociatividad es una estrategia para mejorar los sistemas de producción y comercialización; además de ayudar a mantener estándares de calidad, volúmenes de producción y mercados estables con lo que se puede cubrir la demanda de mercados nacionales e internacionales.

\section{Conclusión}

A la cebada se lo considera un cultivo tradicional por ser un cultivo ancestral y porque en su momento contribuyó a la alimentación básica de la población; sin embargo en la actualidad por la diversificación de alimentos que existen han perdido estas particularidades. Además económicamente no es rentable para las superficies de las fincas que la producen en la provincia del Carchi; mientras que, el cultivo de la frutilla, cultivo no tradicional es de importancia económica para los agricultores de Yaruquí. Fincas menores a una hectárea tienen una utilidad superior a la de 5 ha de cebada en la mitad de tiempo. Para los agricultores estos factores económicos son importantes al elegir un tipo de cultivo, sin considerar otros aspectos de los cultivos tradicionales. Sin embargo, coinciden que la asociatividad es la mejor manera de mejorar sus ingresos, al contar con mayor poder de negociación y disminuir los intermediarios. Se concluye además que en algún momento los cultivos no tradicionales se volverán tradicionales como ocurrió en el cultivo de rosas, que por su importancia económica y social actualmente es considerado tradicional en Ecuador. Se recomienda capacitar a los agricultores para que se organicen y asocien, con el fin mejorar los ingresos de sus miembros.

\section{Literatura citada}

Agronegocios. 2010. Agronegocios.com.ec. Obtenido de Fresas: Su producción y crecimiento. Disponible en http:// agronegociosecuador.ning.com/page/ fresas-su-produccion-y

Cangas, J. 2018. Problemas de la cebada en la Provincia del Carchi. Presidente de la Asociacion de Quinueros del Carchi. El Ángel, Carchi, Ecuador.

ESPAC. 2017. Encuesta de Superficie y Producción Agropecuaria Continua. Disponible en https://www.ecuadorencifras.gob.ec/documentos/web-inec/ Estadisticas agropecuarias/espac/ espac 2017/Informe_Ejecutivo_ES- 
PAC_2017.pdf

Flores, J.F.; Edwin, M.R. 2017. Factores que influyen en la rentabilidad económica de la producción del cultivo de camu camu en la selva peruana. Tzhoecoen 9(1): 94-106.

Hernández, R.; Fernández, C. 2010. Metodología de la Investigación (Quinta ed.). México D.F. McGrawHill.

Iglesias, J.M. 2017. Semillas de colza y cebada: estrategias de mejoramiento y comercialización de nuevos cultivares en Argentina (2004-2014).

IICA. 2017. Instituto Interamericano de Cooperación para la Agricultura. 2017. Manual de Buenas Prácticas Agrícolas y Producción para el Cultivo de la Fresa.

Instituto Interamericano de Cooperación para la Agricultura. San José: Masterlitho. Disponible en http://www.iica. int

Instituto Nacional de Estadísticas y Censos. 2017. Encuesta de superficie y producción agropecuaria continua (ESPAC). Superficie, producción y ventas, según región y provincia, cebada (Grano seco). Quito.

Luna-Morales, C.D.C. 2015. Ciencia, conocimiento tradicional y etnobotánica. Etnobiología, 2(1), 120-136.

Ministerio de Agricultura, Ganadería, Acuacultura y Pesca. 2017. Boletín Agrícola Integral. Zona 5. GuayaquilEcuador

Monteros, A.; Salvador, S. 2015. Panorama agroeconómico del Ecuador una visión del 2015. Quito: Dirección de Análisis y Procesamiento de la Información.

Prefectura del Carchi. 2016. Mapas Provinciales. (D. C. S., Editor) Disponible en www.carchi.gob.ec

Sistema de Investigación sobre la Problemática Agraria. 2013. Sistema Rurales - Urbanos en el $D M Q$. SIPAE. Quito: Gráficas Ayerve C.A.

Tustón Chacón, R.G. 2012. Sistematización de experiencias del cultivo de frutilla, para la sierra norte de Pichincha.
Tesis Pregrado. Quito: Universidad Politécnica Salesiana.

Vaquiro, N. F. 2016. Economía y trabajo en el sector agrícola. FLACSO México. 